\title{
Realidade virtual e estereoscopia no ensino da neuroanatomia e neurocirurgia
}

\author{
Mauro Augusto Tostes Ferreira', Sebastião Nataniel Silva Gusmão², \\ Robert Frederich Spetzler ${ }^{3}$
}

Hospital Unimed, Belo Horizonte, MG, Brasil e Instituto Neurológico Barrow, Phoenix, AZ, Estados Unidos.

\section{RESUMO}

Objetivo: Por motivos diversos, o ensino da neuroanatomia durante a graduação médica e na residência de neurocirurgia é deficitário. Apresentamos a realidade virtual e a estereoscopia como eventuais métodos complementares de ensino à neuroanatomia e neurocirurgia. Método: Diversa gama de conteúdo digital interativo e estereoscópico foi produzida utilizando esterogramas de dissecações anatômicas. Resultados: A realidade virtual tenta melhor elaborar o ensino da neuroanatomia e neurocirurgia. Embora o trabalho verse sobre neuroanatomia e neurocirurgia, esses recursos podem ser empregados em qualquer área médica. Conteúdo anatômico de excelência foi adquirido e armazenado de modo que pôde ser manipulado por programa de realidade virtual e estereoscopia. Conclusão: $A$ realidade virtual e a estereoscopia são ferramentas úteis no ensino e na aprendizagem da neuroanatomia e da neurocirurgia.

\section{PALAVRAS-CHAVE}

Neuroanatomia, neurocirurgia, tecnologia biomédica, educação médica.

\section{ABSTRACT}

Virtual reality and stereoscopy for neuroanatomy and neurosurgery teaching

Objective: Because of numerous factors, neuroanatomy and microneurosurgical anatomy knowledge are insufficient during medical school and medical residency in neurosurgery. We present virtual reality and stereoscopy as eventual complementary teaching tools of neuroanatomy and neurosurgery. Method: A vast array of digital interactive and stereoscopic material has been created based on stereograms of real anatomical dissections. Results: The purpose of virtual reality is try to offer a better and more elaborate means for teaching neuroanatomy and neurosurgery. Although this paper has focused virtual reality and stereoscopy on neuroanatomy and neurosurgery, these tools can be applied to virtually all fields of medicine. An excellent anatomical content has been collected and included in the virtual reality program, using stereoscopy. Conclusion: The virtual reality and stereoscopy are useful learning and teaching tools for neuroanatomy and neurosurgery.

\section{KEYWORDS}

Neuroanatomy, neurosurgery, biomedical technology, medical education.

1 Doutor em Cirurgia pelo Departamento de Cirurgia da Faculdade de Medicina da Universidade Federal de Minas Gerais (UFMG), Neurocirurgião do Hospital Unimed, Belo Horizonte, MG, Brasil.

2 Professor titular de Neurocirurgia da Faculdade de Medicina da UFMG, Belo Horizonte, MG, Brasil.

3 Diretor, Instituto Neurológico Barrow, Phoenix, AZ, Estados Unidos. Professor-Chefe, Universidade do Arizona, Tucson, AZ, Estados Unidos. 


\section{Introdução}

A neuroanatomia é matéria complexa e ensinada de forma deficiente na graduação. A falta de laboratórios de anatomia, a dificuldade de se obter espécimes, bem como o desinteresse sobre o tema perpetuam essa deficiência até a residência de neurocirurgia. Isso é grave, pois o conhecimento profundo da disposição espacial das estruturas do sistema nervoso constitui a base da neurocirurgia.

A percepção visual e a construção da memória humana são estereoscópicas. Assim, a percepção primária das estruturas anatômicas deveria ser estereoscópica. A informática permite transformar dissecações de cadáveres em realidade virtual (RV) estereoscópica que constituem ferramentas úteis complementares de ensino. O objetivo deste trabalho é descrever métodos de RV e estereoscopia, baseados em registros anatômicos como potenciais ferramentas de ensino da neuroanatomia e neurocirurgia.

\section{Materiais e métodos}

\section{Preparo, manipulação, cuidados e dissecação das peças}

Dez segmentos cefálicos cadavéricos foram preparados para dissecação, sendo esta realizada no laboratório de microneuroanatomia do Barrow Neurological Institute $^{\circledR ”}\left(\mathrm{BNI}^{\circledR}\right)$, em Phoenix, AZ, EUA, e no Laboratório de Anatomia Neurocirúrgica da Faculdade de Medicina da UFMG, Belo Horizonte, MG. Os espécimes "frescos" (até 48 horas post-mortem) tiveram as artérias carótidas internas ou comuns, as artérias vertebrais e as veias jugulares internas identificadas e dissecadas na base do pescoço. Tubos plásticos flexíveis foram introduzidos no interior dos vasos e fixados por meio de fio de sutura. Os vasos foram irrigados com solução desodorante e desinfetante (HEXAPhENE MA-37, Party-Boy, S Calton Road, EastLyme, CT, EUA). A seguir, efetuou-se irrigação copiosa com água corrente para remoção de coágulos vasculares. Após esse passo, silicone colorido em vermelho foi injetado na árvore arterial e silicone azul, no sistema venoso. Os espécimes foram deixados submersos em solução embalsamante por dois meses.

\section{Fotogramas estereoscópicos analógicos e digitais}

Fotogramas estereoscópicos consistem em par de imagens do mesmo objeto com ângulos de vista dife- rentes. No início, utilizou-se câmera analógica Nikon F2M (Nikon, Japão), com filme Velvia ${ }^{\circledR} 35$ milímetros e ISO/ASA 100 (Fujifilm, Japan), colocada em tripé de precisão (Manfrotto ${ }^{\circledR}$ Tripod, by Bogen, Itália), de modo a assegurar perfeito posicionamento horizontal ou vertical da câmera. Utilizou-se objetiva Nikon 105 milímetros 1:2.8 D (Nikon, Japão). Posteriormente, câmeras digitais Canon D30 (Canon D30 EOS, Canon Inc., Japão) e Canon D60 (Canon D60 EOS, Canon Inc., Japão) com lente Canon 18-55 milímetros EF-S e Canon Ultrasonic EF (100 mílímetros f/2.8 Macro USM) foram utilizadas. Os fotogramas de imagens múltiplas destinadas à montagem iconográfica do programa RV foram sempre adquiridos a partir das câmeras digitais (duas) acopladas ao microscópio robótico MKM. As câmeras foram cuidadosamente alinhadas para obtenção de estereogramas.

\section{Vídeo digital estereoscópico}

Utilizou-se vídeo digital estereoscópico para demonstrar acessos operatórios e as relações tridimensionais das estruturas envolvidas. Uma câmera filmadora, semelhante às câmeras filmadoras convencionais, foi acoplada entre a objetiva e a extrativa do microscópio (câmera 3D Carl Zeiss, Inc, Alemanha; microscópio OPMICS $^{\text {mw }}$, Carl Zeiss, Alemanha). Óculos especiais (MedLive $^{\circledR}$, Carl Zeiss, Alemanha) foram utilizados para verificar o prosseguimento das dissecações.

\section{Modelos estereoscópicos e programa de realidade virtual}

Todo material registrado em foto ou vídeo foi transferido ao Setor de Publicações do BNI ${ }^{\circledR}$, onde especialistas em computação gráfica produziram modelos anatômicos, assim como os manipularam e criaram efeitos especiais como interação com o conteúdo anatômico. Os modelos estereoscópicos foram criados por programa de computador denominado Maya ${ }^{\circledR}$ (Maya 6.0 Unlimited; Alias System, Tokyo, Japão). Ele permitiu que se criasse qualquer tipo de objeto a partir de formas elementares simples. O Maya ${ }^{\circledR}$ utiliza os três planos ortogonais de modo a se criar modelos tridimensionais, estereoscópicos ou não. Pode-se, ainda, criar camadas superpostas de imagens, de modo a fornecer percepção de transparência ou semitransparência, muito útil quando do estudo de estruturas ósseas, como o osso temporal, particularmente o osso petroso que abriga estruturas importantes. É possível inserir fotogramas, sequências de exames de RM ou de TC, vídeos, sons, entre outros, o que permite realizar e criar gama infinita de situações e simulações por meio de tecnologia digital. 
Além disso, o programa permite criar animações com sequências em que o modelo segue padrão de movimentos preestabelecidos.

O programa realidade virtual (RV), tecnicamente denominado VRML ("Virtual Reality Modeling Langua$g e$ "), é um tipo de formato criado para confeccionar e manipular objetos tridimensionais. Sua interface com o programa Director ${ }^{\circledR}$ possibilitou a "fusão" de imagens de dissecações e aplicação de comandos de mudanças de ângulos de vista via QuickTime ${ }^{\circledR}$. Animações obtidas no Maya ${ }^{\circledR}$ podem ser trabalhadas no Director ${ }^{\circledR}$. O Director $^{\circledR}$ determina o fator tempo a qualquer sequência de eventos que seguem determinada linha temporal.

A "fusão" de diversas imagens forma uma "camada" ou "continuum" convexo de imagens. O tamanho da camada é variável e as imagens que as compõem seguem relação de vizinhança com imagens adjacentes seguindo os princípios das fotos estereoscópicas (Figura 1). Essa camada convexa, também conhecida como grid (composta de fileiras e colunas), foi planificada pelo programador de computador.
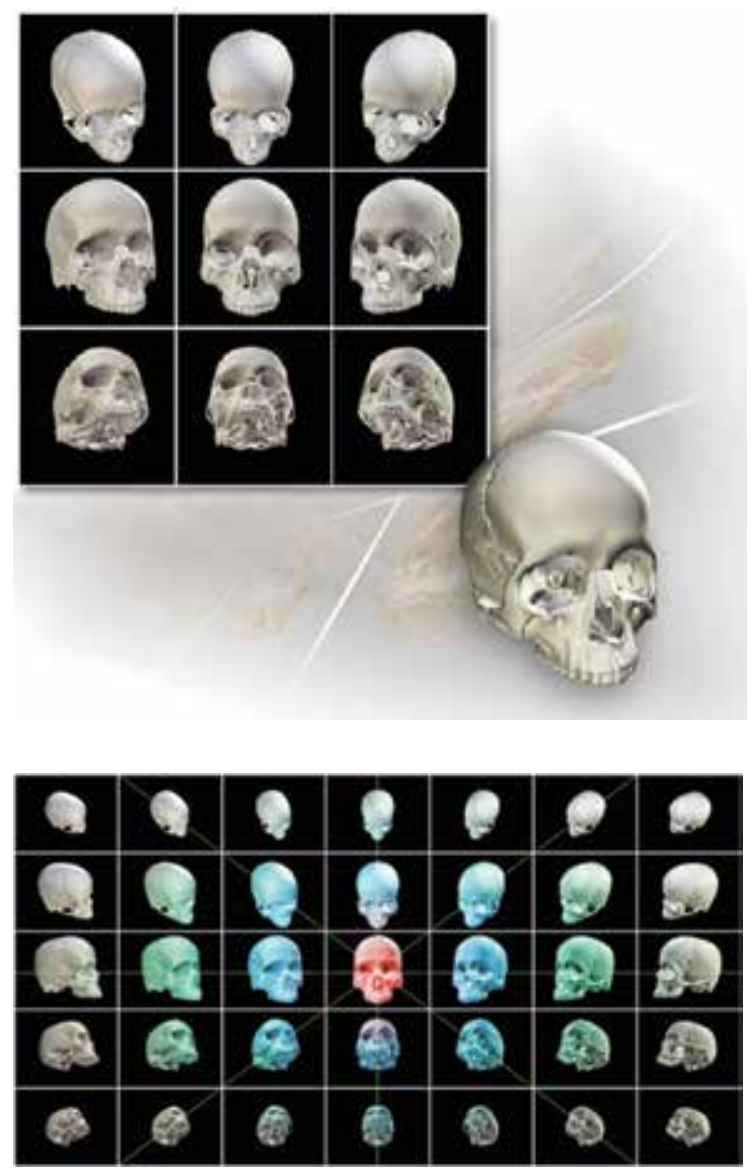

Figura 1 - "Grids" ou camadas de imagens de número variável de fotogramas relacionadas entre si e que permitem visibilização do objeto sob vários ângulos de vista. Acima camada de 9 imagens. Abaixo camada de 35 imagens.
Foi possível desenvolver modelos nos quais, por meio de comandos específicos, os diferentes planos anatômicos puderam ser vistos através de graus variados de transparência, ou a partir de camadas superficiais se obteve acesso a camadas mais profundas, por meio dos diferentes "grids" superpostos. O programa RV foi inserido no Adobe Director ${ }^{\circledR}$ (Adobe Director MS, 2004) que possui esse nome devido ao fato de possibilitar, a quem o manuseia, sensação de poder "dirigir" ou "manipular" a sequência de ações desejada.

O Adobe Director ${ }^{\circledR}$, o Maya ${ }^{\circledR}$ e o Adobe AfterEffects ${ }^{\circledR}$ possibilitam que objetos $3 \mathrm{D}$ sejam introduzidos, manipulados e mostrados de diferentes maneiras. A combinação dos programas QuickTime ${ }^{\circledR}$ e RV criou a interface QTVR ("Adobe QuickTime Virtual Reality $\left.{ }^{\circledR ”}\right)$ onde se pode inserir conteúdo multimídia, de modo a se criar ambiente digital virtual que possibilita interação entre diversos tipos de mídia. Utilizou-se o Apple QuickTime ${ }^{\circledR}$ para “ativar" os comandos predeterminados pelo Maya ${ }^{\circledR}$, Director ${ }^{\circledR}$ e AfterEffects ${ }^{\circledR}$. As animações estereoscópicas foram produzidas por meio do programa Adobe AfterEffects ${ }^{\circledR}$. O conteúdo digital entrelaçado é o método de eleição para produzir conteúdo estereoscópico, pois preserva cores, mas anáglifos vermelhos e cianos ou amarelo-âmbar e azuis também foram empregados devido à possibilidade de impressão de figuras em papel. Imagens entrelaçadas não podem ser impressas. Utilizaram-se dois computadores de mesa para confecção de modelos, animações e RV.

\section{Resultados}

A técnica de embalsamento se mostrou eficaz. Foram preparados encéfalos rígidos, propícios a secções anatômicas e tecidos extracranianos maleáveis, passíveis de retração. Quando se requereu retração encefálica, ela ocorreu de modo lento e gradual ( $\mathrm{Fi}$ gura $2 \mathrm{~A}$ e B).

\section{Fotos estereoscópicas analógicas e digitais}

Foram obtidos aproximadamente sete mil pares estereoscópicos. Observou-se nítida curva de aprendizado em relação às técnicas de fotografia. Os estereogramas constituíram a base do banco de dados para confecção de animações, simulações, assim como para a composição de modelos de realidade virtual (RV). Mesmo estereogramas simples de crânio proporcionaram imagens "atrativas" (Figura 3A e B). 
A

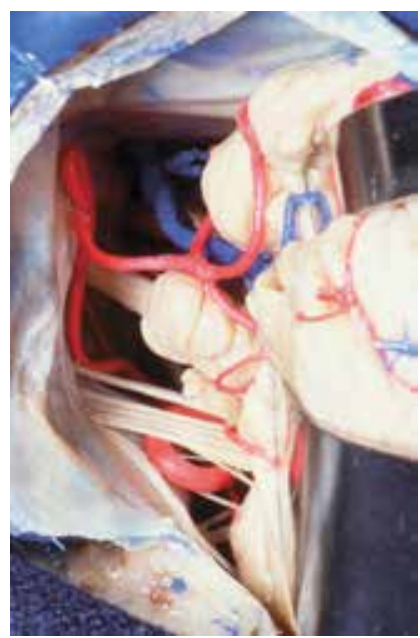

B

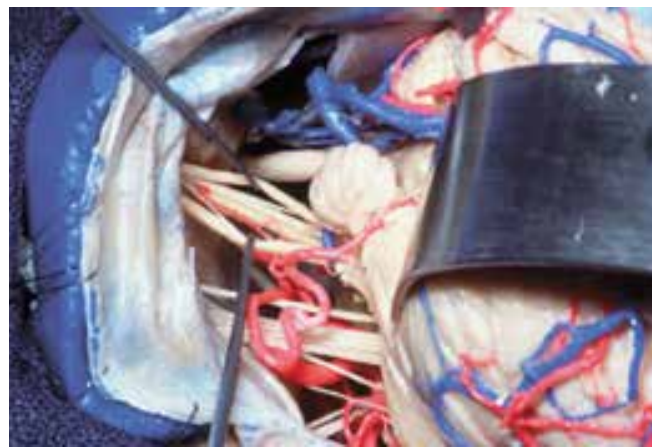

Figura 2 ( A e B) - Esposição das estruturas anatômicas no ângulo ponto-cerebelar. Devido à rigidez dos encéfalos, a retração tecidual se deu de modo lento e gradual.

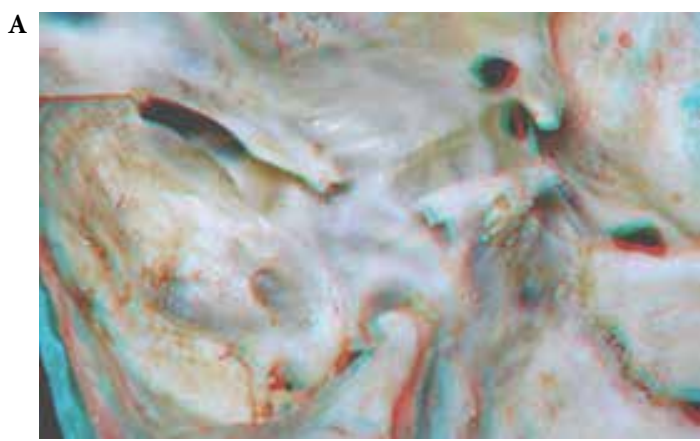

B

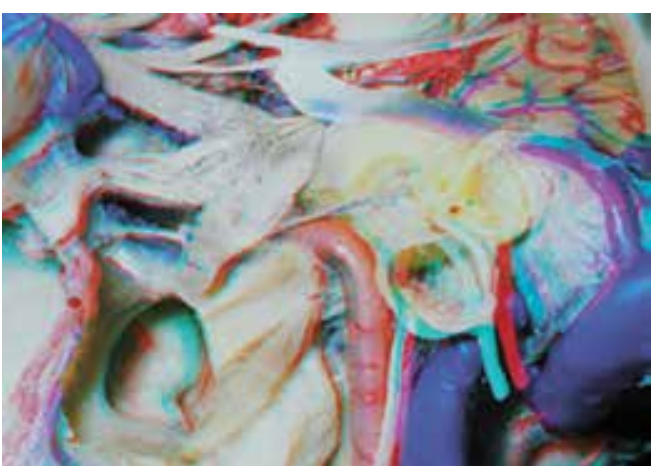

Figura 3 - Estereogramas anáglifos vermelhos-ciano. (A) Vista superolateral esquerda da base do crânio com câmera analógica.

(B) Vista de dissecção da fossa média, infratemporal e espaço para-faríngeo anterior esquerdo com câmera digital.
Raramente se utilizou microscópio para obter imagens analógicas, pois utilizou-se teleconversor. No entanto, o microscópio foi utilizado de modo frequente para fotos digitais. Duas câmeras foram acopladas ao microscópio, precisamente niveladas em relação ao eixo horizontal. As séries de fotos que compuseram as "camadas de fotos" (ou "grids") para o projeto RV foram sempre obtidas a partir de duas câmeras acopladas ao microscópio robótico MKM.

Foram obtidas imagens estereoscópicas utilizando anáglifos vermelhos e cianos, âmbar-amarelado e azuis, e imagens entrelaçadas. Os estereogramas entrelaçados forneceram as melhores imagens, pois não subtraem cor. No entanto, não é possível imprimir estereogramas entrelaçados. Já anáglifos podem ser facilmente impressos. Há, no entanto, distorção de cores quando se imprimem anáglifos. $\mathrm{O}$ monitor do computador utiliza o sistema RGB (vermelho, verde, azul). Já as impressoras utilizam sistema CMYK (ciano, magenta, amarelo, preto). Esses padrões são universais.

\section{Vídeo estereoscópico}

Inúmeros vídeos estereoscópicos de dissecações cadavéricas foram adquiridos. De modo simultâneo, vídeos estereoscópicos foram obtidos no centro operatório (Figura 4).

\section{Animações estereoscópicas e não estereoscópicas}

Grande número de animações foi criado. Algumas animações, mais longas, não foram geradas com estereoscopia, o que aumentaria em demasia o volume do conteúdo digital. Outras, essencialmente autoexplicativas, também foram concebidas em duas dimensões. Todas as demais, principalmente as que demonstraram acidentes anatômicos e técnica operatória, foram concebidas com técnica estereoscópica (Figura 5).

\section{Realidade virtual estereoscópica interativa}

Obtiveram-se ótimos exemplos do programa RV, criados de modo a permitir interação com o expectador, configurando, assim, a RV interativa, ou QTVR (“QuickTime Virtual Reality"). Obtiveram-se registros de várias áreas do cérebro e base do crânio. Utilizou-se camada, na maioria das vezes, contendo 16 imagens para vista microscópica e camada de extensão variada para vista macroscópica. Estereoscopia foi aplicada a todos os QTVR. Por meio de comandos específicos, o expectador pôde interagir com a anatomia exposta de modo a mimetizar o movimento do microscópio 


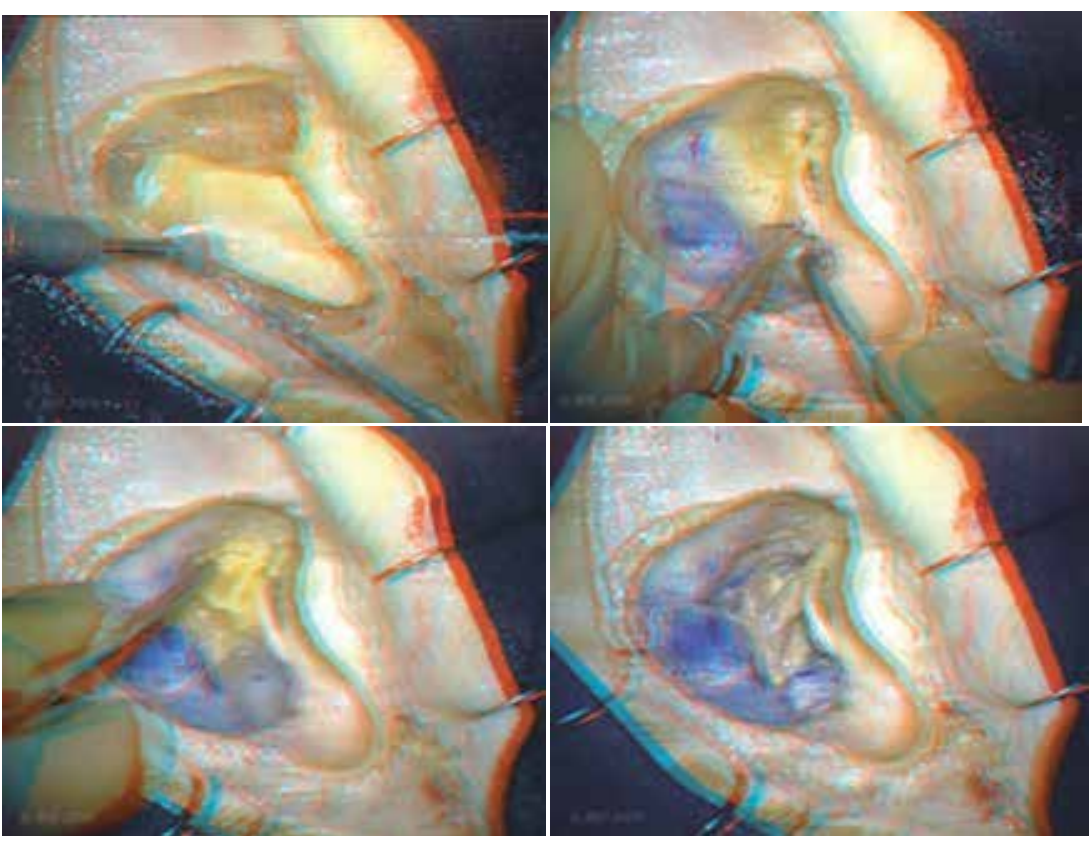

Figura 4 - Fotogramas estereoscópicos anáglifos vermelhos-ciano obtidos a partir de vídeo estereoscópico com demonstração do acesso translabiríntico ao ângulo ponto-cerebelar.
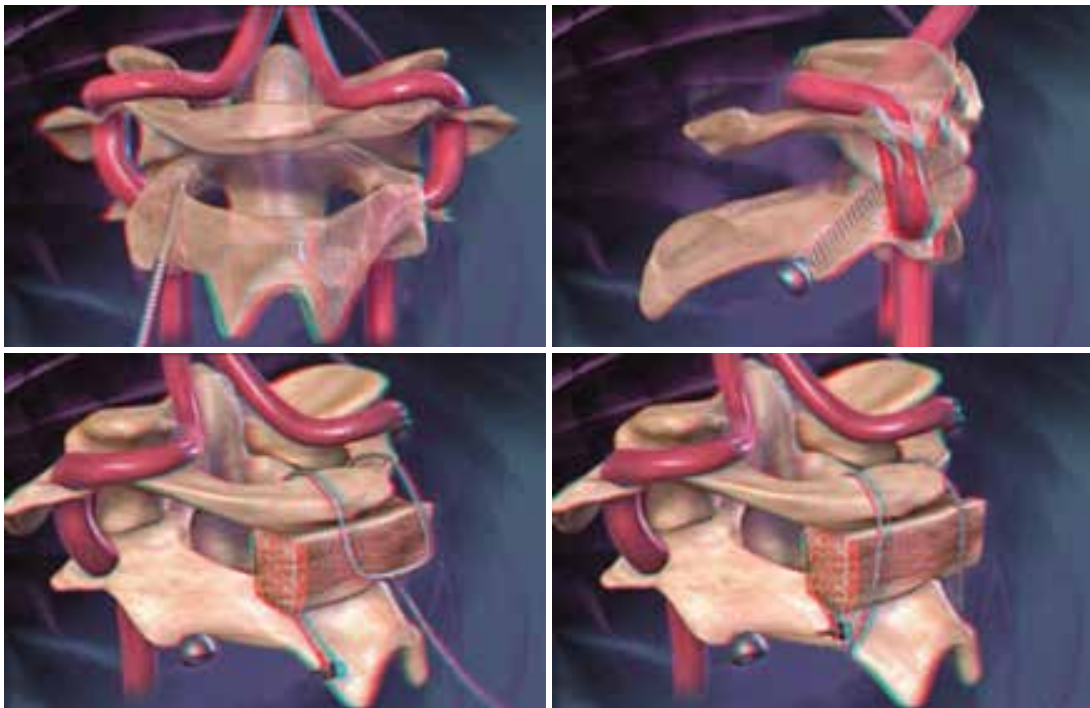

Figura 5 - Sequência de fotos estereoscópicas anáglifas vermelhas-ciano obtidas a partir de animação digital que demonstra os diferentes passos de uma das técnicas de fixação C1-C2.

operatório. Todos os ângulos de interesse de uma determinada exposição operatória puderam ser vistos (Figuras 6 e 7). Obtiveram-se sempre vista panorâmica e microscópica da mesma região. Modelo semitransparente estereoscópico interativo contendo as estruturas neurovasculares das três superfícies do osso temporal foi criado, mas é exemplificado por meio de imagens planas. Por motivo desconhecido, sua impressão em papel não fez gerar imagem estereoscópica.
Às imagens virtuais estereoscópicas, associaram-se vídeos estereoscópicos, animações estereoscópicas e estereogramas, de modo que os diferentes métodos de estereoscopia forneceram rico material de ensino de anatomia e técnica neurocirúrgica. Utilizou-se ainda conteúdo multimídia (ilustrações esquemáticas, narrações e textos sobre os temas), de maneira que o usuário pudesse escolher o modo e a sequência do método de aprendizado a ele oferecido. 

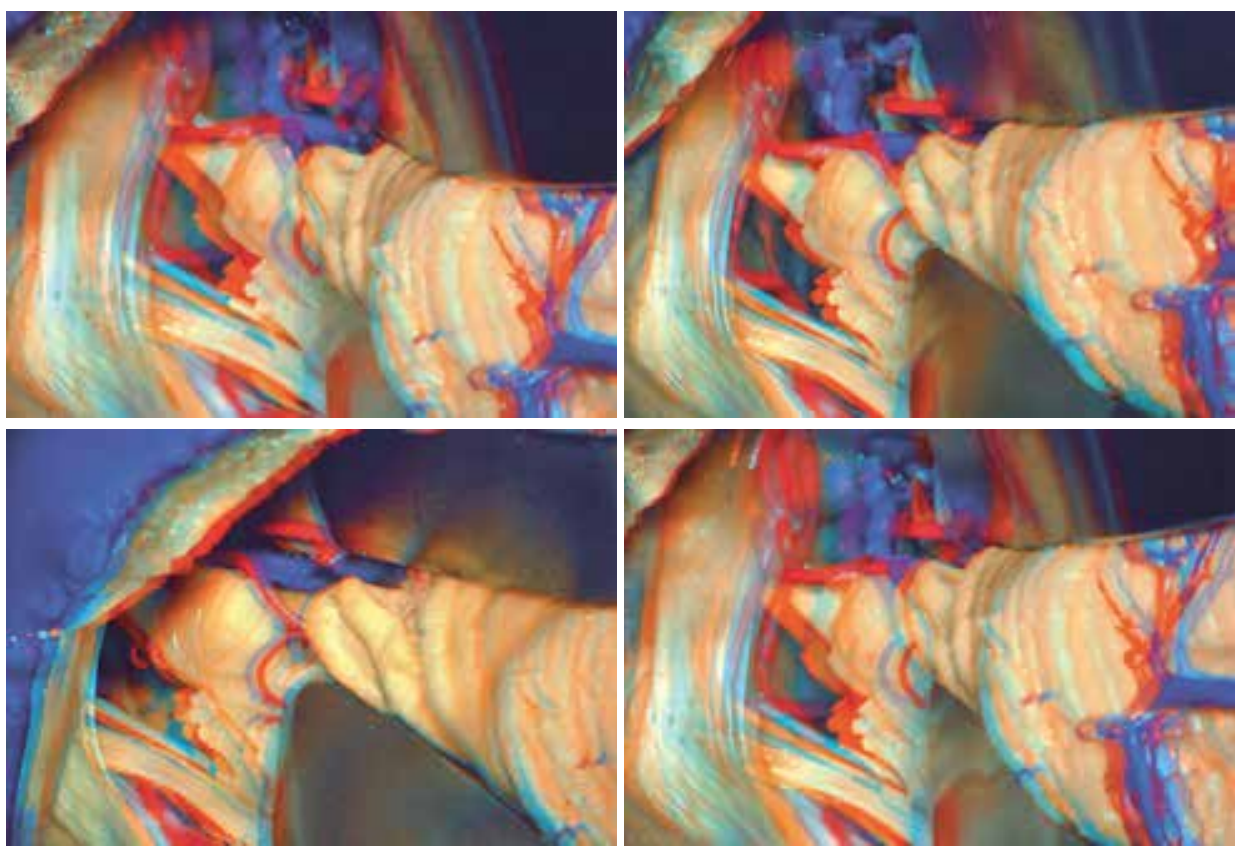

Figura 6 - Quatro fotos estereoscópicas anáglifas vermelho-ciano de sequência QTVR mostrando as diferentes estruturas do ângulo ponto-cerebelar sob diferentes ângulos de vista. A sequência de 16 imagens pode ser "manipulada" utilizando "comandos" específicos.
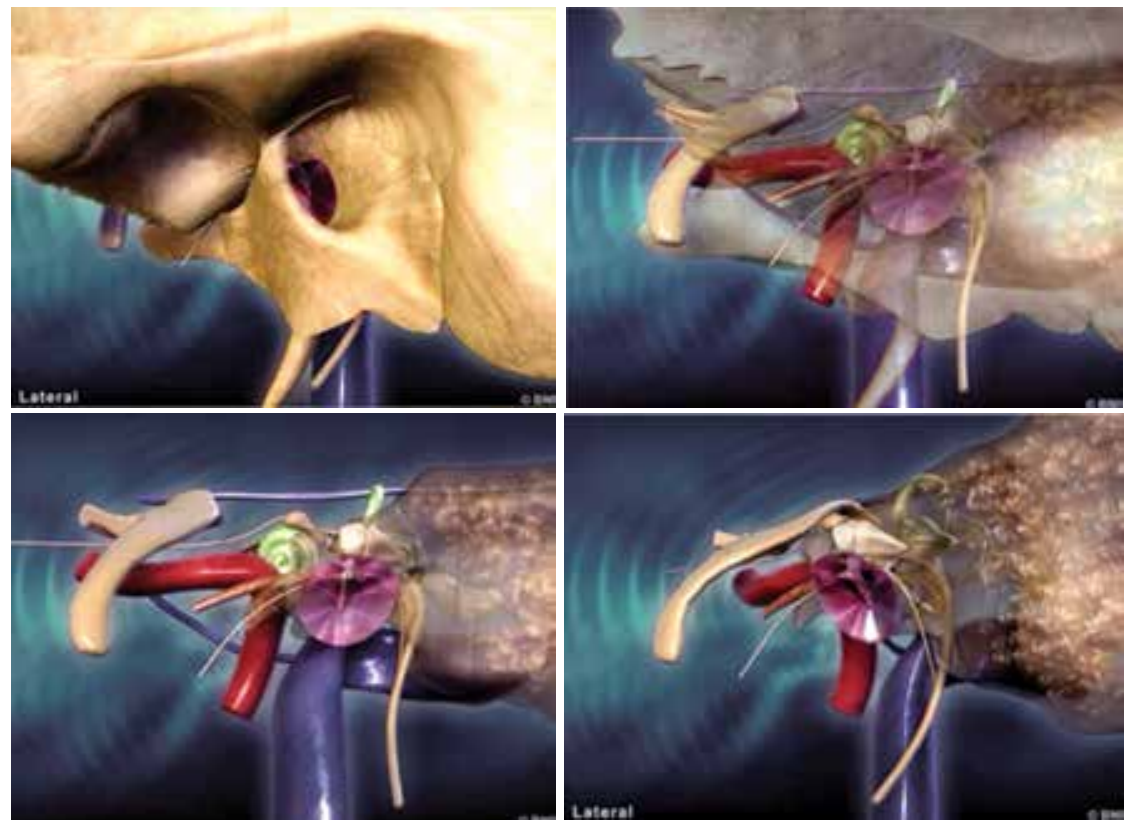

Figura 7 - Estruturas do osso temporal. Sequência de fotogramas que mostra o osso temporal e seu interior. Este modelo permite que o osso se torne semitransparente ou transparente e que as várias estruturas sejam vistas sob diferentes ângulos. $O$ expectador interage com o modelo e estuda, de várias maneiras, as relações anatômicas tridimensionais. Este modelo com vista estereoscópica também foi criado. 


\section{Discussão}

\section{Início e evolução do conhecimento da neuroanatomia e utilização da estereoscopia}

A evolução do conhecimento se deveu à melhor representação visual do que se observava. Vários trabalhos verificados na literatura utilizaram a estereoscopia com múltiplos propósitos: de ensino ${ }^{1-8}$; treinamento ${ }^{9-15}$; planejamento operatório ${ }^{16-21}$; tratamento ${ }^{22-27}$ e documentação. ${ }^{28-30}$

Considerou-se, neste trabalho, a estereoscopia como componente essencial dos ambientes de realidade virtual (RV). Vesalius, há cinco séculos, observou, dissecou, compreendeu e realizou registros pictóricos da anatomia. Esse método não é utilizado hoje na maioria das faculdades de medicina, e a anatomia macroscópica por ele descrita é desconhecida da maior parte dos estudantes. ${ }^{31,32}$

Neste trabalho, procurou-se ilustar a anatomia por meio de estereogramas de dissecações detalhadas. Aproximadamente sete mil pares estereoscópicos foram obtidos, o que serviu de base para a compreensão tridimensional das estruturas anatômicas e posteriormente para utilização em programas de computador. Cinco estudos se basearam em dissecações cadavéricas para criar modelos anatômicos. Três deles relataram modelos interativos estereoscópicos baseados em registro anatômico estereoscópico. ${ }^{1,2,5}$ Outro estudo registrou, por meio de estereogramas, áreas anatômicas expostas durante operação. ${ }^{3} \mathrm{O}$ quinto estudo, realizado na Universidade da Flórida ${ }^{33}$, baseou-se em dissecações precisas para gerar modelo tridimensional interativo não estereoscópico. O pilar do presente estudo é o trabalho publicado por Henn et al. ${ }^{1}$ Diferiu dos demais por aplicar estereoscopia a grande quantidade de fotogramas, animações, modelos interativos, vídeos de dissecações e de operações, e em programas de RV. Modelos estereoscópicos com camadas semitransparentes se mostraram úteis em auxiliar compreensão visual de estruturas complexas como o tronco encefálico e o osso petroso. Não encontramos, na literatura, confecção de tal tipo de modelo e acreditamos termos sido os primeiros a desenvolvê-los e apresentá-los.

\section{Ensino atual da neuroanatomia}

Leciona-se neuroanatomia em curto período de tempo. A dissecação foi praticamente abandonada. Esse processo se deve a: progressiva escassez de espécimes anatômicos; tempo reduzido para se dedicar a assunto assaz complexo; depreciação da anatomia como matéria fundamental em medicina; desenvolvimento de outras áreas da medicina; falsa percepção da não necessidade de conhecimento anatômico devido ao desenvolvimento de técnicas sofisticadas de obtenção de imagens; progressiva falta de instrutores qualificados. ${ }^{31}$ Entre esses, considera-se grave e irreversível escassez de espécimes e de tempo para se dedicar ao estudo da anatomia.

\section{Início da residência médica em neurocirurgia e seu ensino atual}

O conhecimento insuficiente da neuroanatomia perdura até a residência médica de neurocirurgia. Importante método de aprendizado envolve livros-texto da matéria. Apesar de importantes fontes de informação e referência, livros não se constituem em ferramentas de aquisição de habilidade operatória ou de extrapolação mental tridimensional. O laboratório de microneuroanatomia cirúrgica é o local mais apropriado para treinamento e aprendizado, porém há poucos laboratórios bem equipados disponíveis no mundo.

\section{Realidade virtual: início e emprego em neuroanatomia e neurocirurgia}

O termo realidade virtual (RV) foi introduzido na década de 1960, com intuito de criar simulação de situações perigosas sem risco para o indivíduo ou para os que o circundam. A indústria bélica foi a primeira a utilizar simuladores de voo e, em seguida, simuladores de aviação comercial foram introduzidos. ${ }^{34} \mathrm{~A}$ telemedicina surgiu devido à necessidade de operação de soldados em campos de batalha a partir de local seguro, assim como resolução de problemas de saúde de astronautas situados na órbita do planeta. ${ }^{35}$

A RV tem despertado crescente interesse em medicina principalmente nas diversas áreas da cirurgia. Na medicina atual, em que a tolerância a erro é cada vez mais baixa, treinamento de situações de risco em ambientes virtuais ou de simulação é necessidade premente. A necessidade de complementação do método halstediano é clara e a RV pode fornecer valiosa contribuição. ${ }^{36}$ Um estudo relatou que, antes do advento da restrição de horas de trabalho na residência médica, o residente de neurocirurgia inglês dedicava 30 mil horas ao aprendizado e hoje dedica 15 mil horas. Astronauta da National Aeronautics and Space Administration (NASA) dedica 10 mil horas a treinamento específico e piloto comercial dedica 5 mil horas para que possa conduzir avião. ${ }^{37} \mathrm{Em} 2003$, Kelly ${ }^{38}$ fez críticas incisivas em relação à precária situação da RV em neurocirurgia. Embora o neurocirurgião manipule a matéria mais elaborada do 
universo - o cérebro humano -, pouca contribuição se observou para criação de ambientes virtuais de treinamento, ao passo que milhões de dólares são gastos em simulação aeronáutica, bélica e aeroespacial, pois nesses casos acidentes causam "catástrofes". Em operações, ocasionam morte ou "apenas sequela". ${ }^{38}$

A RV despertou real interesse em medicina nas duas últimas décadas. O número de publicações aumentou exponencialmente após o regime de restrição de horas nos programas de residência médica nos Estados Unidos e na Europa, mas pode-se inferir que o desenvolvimento exponencial da informática também pode explicar, em parte, essa observação.

O conceito de RV em neurocirurgia é impreciso. Análise da literatura mostrou que se enquadram nesse conceito modelos tridimensionais estereoscópicos ou não, manipuláveis ou não. Vários graus de imersão e interação sensorial com retorno háptico, auditivo e vestibular foram propostos. Em toda a revisão da literatura, encontrou-se apenas um estudo que tenta definir a RV em neurocirurgia, porém há nítida sobreposição com componentes de inteligência artificial. ${ }^{34}$

Esses autores, do grupo da Universidade do Sul da Califórnia, consideraram toda gama possível de interação sensorial com o ambiente criado por computador como indispensável para se caracterizar $\mathrm{RV}^{34}$ Assim, a proposição de utilização de aparelhos complexos foi descrita. ${ }^{9}$ De acordo com a classificação dos autores, a grande maioria dos estudos, incluindo o que aqui se apresenta, enquadraria-se no conceito de "RV simplificada". Esses autores propõem utilização de modelos complexos, caros e, no mais das vezes, de caráter experimental. Enfatizam a necessidade de retorno háptico preciso, o que, como se mostra no presente, irreal. De modo paradoxal, os mesmos autores publicam estudo que corrobora essa opinião. ${ }^{34}$

Há esboço de RV como método de treinamento de ventriculostomias. ${ }^{11,12,39,40} \mathrm{~A}$ RV também já é citada como uma ferramenta no processo de planejamento operatório. ${ }^{13,16-21,24,41-45}$ A planificação de ato operatório por cirurgião experiente e a compreensão do que se fará, por parte do aprendiz, utilizando-se métodos visuais atrativos, principalmente se aliados à estereoscopia, podem fornecer meio preciso e rápido de aprendizado. A RV se presta a planificar operações de modo a utilizar melhores ângulos de vista com menor risco para o paciente. Destaca-se o estudo de Rosahl et al ${ }^{46}$ que propõe uso de modelos tridimensionais estereoscópicos semitransparentes, gerados a partir de exames de RM do paciente, que demonstra características anatômicas individuais e as relações de estruturas normais e a afecção. Pelo fato de as imagens conterem fiduciais, esse modelo se mostrou mais útil que as imagens triplanares convencionais do neuro- navegador. A possibilidade de ensino e extrapolação tridimensional a partir de exames de imagens de RM pode ser ferramenta útil ao aprendiz.

A RV interativa como fonte de ensino já é citada como ferramenta útil. Modelos precisos do encéfalo e da base do crânio, e especialmente aqueles baseados em imagens de dissecações reais, mostraram-se úteis. ${ }^{1,2,5,33}$ A RV forneceu ainda ambientes de simulação. ${ }^{2,9,39,47-53}$

O presente estudo teve como objetivo criar método de RV com intuito didático. Não se utilizou retorno háptico, pois acredita-se que essa modalidade de interação sensorial ainda esteja longe de ser alcançada. Possibilitou-se, no entanto, oportunidade de interação com o conteúdo digital gerado. Os movimentos do microscópio operatório puderam ser mimetizados quando o aluno interagiu com camadas de imagens que proporcionaram vista estereoscópica panorâmica de várias regiões anatômicas. Foram utilizados programas de computador comercializados e de fácil aquisição (Maya $^{\circledR}$, Adobe Director ${ }^{\circledR}$, Adobe AfterEffects ${ }^{\circledR}$, QuickTime Virtual Reality ${ }^{\circledR}$ ), em contraste com outros autores que descreveram "softwares" personalizados e complexos. Assinala-se, no entanto, a necessidade de "experts" em computação gráfica para manuseio dos programas. ${ }^{54}$ Todo o material aqui criado pode ser enviado a qualquer outro computador munido de programas básicos de reprodução gráfica digital. Quanto a RV com retorno háptico, espera-se que possa se constituir em ferramenta útil para auxiliar o treinamento da neurocirurgia em algum tempo futuro.

\section{Perspectivas futuras}

Estudo que defina de modo preciso a RV e seus componentes é necessário para que possa haver homogeneidade de nomenclatura e comparação de resultados. Espera-se que, em futuro próximo, sua validação como método complementar de ensino da neuroanatomia e neurocirurgia e de planificação em neurocirurgia seja alcançado. A validação como método de treinamento deverá ocorrer em futuro mais distante.

\section{Conclusão}

As técnicas de realidade virtual e estereoscopia baseadas em imagens de dissecações de peças anatômicas podem ser ferramentas complementares úteis no ensino da neuroanatomia e neurocirurgia. 


\section{Agradecimento}

Os autores agradecem a Fundação de Amparo à Pesquisa do Estado de Minas Gerais (Fapemig) pelo apoio financeiro dado a esta pesquisa.

\section{Conflito de interesses}

Os autores declaram inexistência de conflito de interesses na realização deste trabalho.

\section{Referências}

1. Henn JS, Lemole GM Jr, Ferreira MA, Gonzalez LF, Schornak M, Preul MC, et al. Interactive stereoscopic virtual reality: a new tool for neurosurgical education. Technical note. J Neurosurg. 2002;96(1):144-9.

2. Bernardo A, Preul MC, Zabramski JM, Spetzler RF. A three-dimensional interactive virtual dissection model to simulate transpetrous surgical avenues. Neurosurgery. 2003;52(3):499-505.

3. Balogh A, Preul MC, Schornak M, Hickman M, Spetzler RF. Intraoperative stereoscopic QuickTime Virtual Reality. J Neurosurg. 2004;100(4):591-6.

4. Rubino PA, Rhoton AL Jr, Tong X, Oliveira Ed. Threedimensional relationships of the optic radiation. Neurosurgery. 2005;57(Suppl 4):219-27.

5. Balogh AA, Preul MC, László K, Schornak M, Hickman M, Deshmukh $\mathrm{P}$, et al. Multilayer image grid reconstruction technology: four-dimensional interactive image reconstruction of microsurgical neuroanatomic dissections. Neurosurgery. 2006;58(Suppl 1):ONS157-65.

6. Anatomy and surgical approaches of the temporal bone and adjacent areas. Neurosurgery. 2007;61(Suppl 4):1-250.

7. Smith DM, Oliker A, Carter CR, Kirov M, McCarthy JG, Cutting CB. A virtual reality atlas of craniofacial anatomy. Plast Reconstr Surg. 2007;120(6):1641-6.

8. Fernández-Miranda JC, Rhoton AL Jr, Alvarez-Linera J, Kakizawa Y, Choi C, de Oliveira EP. Three-dimensional microsurgical and tractographic anatomy of the white matter of the human brain. Neurosurgery. 2008;62(6 Suppl 3):989-1026.

9. Spicer MA, van Velsen M, Caffrey JP, Apuzzo ML. Virtual reality neurosurgery: a simulator blueprint. Neurosurgery. 2004;54(4):783-97.

10. Wang P, Becker AA, Jones IA, Glover AT, Benford SD, Greenhalgh CM, et al. A virtual reality surgery simulation of cutting and retraction in neurosurgery with force-feedback. Comput Methods Programs Biomed. 2006;84(1):11-8.

11. Banerjee PP, Luciano CJ, Lemole GM Jr, Charbel FT, Oh MY. Accuracy of ventriculostomy catheter placement using a head- and hand-tracked high-resolution virtual reality simulator with haptic feedback. J Neurosurg. 2007;107(3):515-21.

12. Lemole GM Jr, Banerjee PP, Luciano C, Neckrysh S, Charbel FT. Virtual reality in neurosurgical education: part-task ventriculostomy simulation with dynamic visual and haptic feedback. Neurosurgery. 2007;61(1):142-8.
13. Wong GK, Zhu CX, Ahuja AT, Poon WS. Craniotomy and clipping of intracranial aneurysm in a stereoscopic virtual reality environment. Neurosurgery. 2007;61(3):564-8.

14. Zirkle M, Roberson DW, Leuwer R, Dubrowski A. Using a virtual reality temporal bone simulator to assess otolaryngology trainees. Laryngoscope. 2007;117(2):258-63.

15. Sharar SR, Miller W, Teeley A, Soltani M, Hoffman HG, Jensen MP, et al. Applications of virtual reality for pain management in burn-injured patients. Expert Rev Neurother. 2008;8(11):1667-74.

16. Anil SM, Kato Y, Hayakawa M, Yoshida K, Nagahisha $\mathrm{S}$, Kanno T. Virtual 3-dimensional preoperative planning with the dextroscope for excision of a 4thventricular ependymoma. Minim Invasive Neurosurg. 2007;50(2):65-70.

17. Kikinis R, Gleason PL, Moriarty TM, Moore MR, Alexander E 3rd, Stieg PE, et al. Computer-assisted interactive three-dimensional planning for neurosurgical procedures. Neurosurgery. 1996;38(4):640-9.

18. Kockro RA, Serra L, Tseng-Tsai Y, Chan C, Yih-Yian S, GimGuan C, et al. Planning and simulation of neurosurgery in a virtual reality environment. Neurosurgery. 2000;46(1):118-35.

19. Kockro RA, Stadie A, Schwandt E, Reisch R, Charalampaki $\mathrm{C}, \mathrm{Ng} \mathrm{I}$, et al. A collaborative virtual reality environment for neurosurgical planning and training. Neurosurgery. 2007;61(5 Suppl 2):379-91.

20. Parikh M, Rasmussen M, Brubaker L, Salomon C, Sakamoto $\mathrm{K}$, Evenhouse R, et al. Three dimensional virtual reality model of the normal female pelvic floor. Ann Biomed Eng. 2004;32(2):292-6.

21. Schutyser F, Poorten VV, Van Cleynenbreugel J, Delaere $P$, Suetens P. An image-based 3D planning environment for hemicricolaryngectomy and reconstruction by tracheal utotransplantation. Comput Aided Surg. 2000;5(3):166-74.

22. Riva G. Virtual reality in neuroscience: a survey. Stud Health Technol Inform. 1998;58:191-9.

23. Lim MW, Burt G, Rutter SV. Use of three-dimensional animation for regional anaesthesia teaching: application to interscalene brachial plexus blockade. $\mathrm{Br} \mathrm{J}$ Anaesth. 2005;94(3):372-7.

24. Stadie AT, Kockro RA, Reisch R, Tropine A, Boor S, Stoeter $\mathrm{P}$, et al. Virtual reality system for planning minimally invasive neurosurgery. Technical note. J Neurosurg. 2008;108(2):382-94.

25. Vasilyev NV, Novotny PM, Martinez JF, Loyola H, Salgo IS, Howe RD, et al. Stereoscopic vision display technology in real-time three-dimensional echocardiography-guided intracardiac beating-heart surgery. J Thorac Cardiovasc Surg. 2008;135(6):1334-41.

26. Bell KM, Frazier EC, Shively CM, Hartman RA, Ulibarri JC, Lee JY, et al. Assessing range of motion to evaluate the adverse effects of ill-fitting cervical orthoses. Spine J. 2009;9(3):225-31.

27. Mirelman A, Bonato P, Deutsch JE. Effects of training with a robot-virtual reality system compared with a robot alone on the gait of individuals after stroke. Stroke. 2009;40(1):169-74.

28. Ribas GC, Bento RF, Rodrigues AJ Jr. Anaglyphic threedimensional stereoscopic printing: revival of an old method for anatomical and surgical teaching and reporting. $J$ Neurosurg. 2001;95(6):1057-66.

29. Shimizu S, Tanaka R, Rhoton AL Jr, Fukushima Y, Osawa $\mathrm{S}$, Kawashima M, et al. Anatomic dissection and classic three-dimensional documentation: a unit of education for neurosurgical anatomy revisited. Neurosurgery. 2006;58(5):E1000.

30. Brack CD, Kessel IL. Evaluating the clinical utility of stereoscopic clinical photography. Stud Health Technol Inform. 2008;132:42-4. 
31. Cottam WW. Adequacy of medical school gross anatomy education as perceived by certain postgraduate residency programs and anatomy course directors. Clin Anat. 1999;12(1):55-65.

32. McCuskey RS, Carmichael SW, Kirch DG. The importance of anatomy in health professions education and the shortage of qualified educators. Acad Med. 2005;80(4):349-51.

33. Kakizawa $\mathrm{Y}$, Hongo $\mathrm{K}$, Rhoton AL Jr. Construction of a three-dimensional interactive model of the skull base and cranial nerves. Neurosurgery. 2007;60(5):901-10.

34. Spicer MA, Apuzzo ML. Virtual reality surgery: neurosurgery and the contemporary landscape. Neurosurgery. 2003;52(3):489-97.

35. Tronnier VM, Staubert A, Bonsanto MM, Wirtz CR, Kunze S. [Virtual reality in neurosurgery]. Radiologe. 2000;40(3):211-7.

36. Rehrig ST, Powers K, Jones DB. Integrating simulation in surgery as a teaching tool and credentialing standard. J Gastrointest Surg. 2008;12(2):222-33.

37. Vloeberghs $M$, Glover A, Benford S, Jones A, Wang P, Becker A. Virtual neurosurgery, training for the future. $\mathrm{Br} J$ Neurosurg. 2007;21(3):262-7.

38. Kelly PJ. Comment. Neurosurgery. 2003;52:496.

39. Phillips NI, John NW. Web-based surgical simulation for ventricular catheterization. Neurosurgery. 2000;46(4):933-6.

40. Lee CK, Tay LL, Ng WH, Ng I, Ang BT. Optimization of ventricular catheter placement via posterior approaches: a virtual reality simulation study. Surg Neurol. 2008;70(3):274-7.

41. Clayman RV. Virtual endoscopy for planning and simulation of minimally invasive neurosurgery. J Urol. 1999;162(5):1875-6.

42. Kawamata $\mathrm{T}$, Iseki $\mathrm{H}$, Shibasaki T, Hori T. Endoscopic augmented reality navigation system for endonasal transsphenoidal surgery to treat pituitary tumors: technical note. Neurosurgery. 2002;50(6):1393-7.

43. Cusimano MD. Virtual reality surgery: neurosurgery and the contemporary landscape a three-dimensional interactive virtual dissection model to simulate transpetrous surgical avenues. Neurosurgery. 2003;53(4):1010-1.

44. Raabe A, Beck J, Rohde S, Berkefeld J, Seifert V. Threedimensional rotational angiography guidance for aneurysm surgery. J Neurosurg. 2006;105(3):406-11.
45. D'Ambrosio AL, Mocco J, Hankinson TC, Bruce JN, van Loveren HR. Quantification of the frontotemporal orbitozygomatic approach using a three-dimensional visualization and modeling application. Neurosurgery. 2008;62(3 Suppl 1):251-60.

46. Rosahl SK, Gharabaghi A, Hubbe U, Shahidi R, Samii M. Virtual reality augmentation in skull base surgery. Skull Base. 2006;16(2):59-66.

47. Zamorano L, Jiang Z, Kadi AM. Computer-assisted neurosurgery system: Wayne State University hardware and software configuration. Comput Med Imaging Graph. 1994;18(4):257-71.

48. Wagner A, Ploder O, Enislidis G, Truppe M, Ewers R. Virtual image guided navigation in tumor surgery--technical innovation. J Craniomaxillofac Surg. 1995;23(5):217-3.

49. Riegel T, Alberti O, Retsch R, Shiratori V, Hellwig D, Bertalanffy $H$. Relationships of virtual reality neuroendoscopic simulations to actual imaging. Minim Invasive Neurosurg. 2000;43(4):176-80.

50. Agus M, Giachetti A, Gobbetti E, Zanetti G, Zorcolo A, John NW, et al. Mastoidectomy simulation with combined visual and haptic feedback. Stud Health Technol Inform. 2002;85:17-23.

51. Li Y, Brodlie K, Phillips N. Web-based VR training simulator for percutaneous rhizotomy. Stud Health Technol Inform. 2000;70:175-81.

52. Acosta E, Muniz G, Armonda R, Bowyer M, Liu A. Collaborative voxel-based surgical virtual environments. Stud Health Technol Inform. 2008;132:1-3.

53. Solyar A, Cuellar H, Sadoughi B, Olson TR, Fried MP. Endoscopic Sinus Surgery Simulator as a teaching tool for anatomy education. Am J Surg. 2008;196(1):120-4.

54. Berndt C, Gheorghian P, Harrington J, Harris A, Mcginnis J. Learning Maya 6. Alias Systems, Toronto, Canada; 2004.

Endereço para correspondência:

Sebastião Nathaniel Silva Gusmão

Rua Padre Rolim, 921-2

Bairro Santa Efigênia

30130-090 - Belo Horizonte, MG, Brasil

E-mail: sebastiaogusmao@gmail.com 